
\title{
$\varepsilon_{\sin }$
}

'Department of Pulmonology and Tuberculosis,

Diakonessenhuis Utrecht, Utrecht, The Netherlands ${ }^{2}$ Department of Medical Microbiology and Immunology, Diakonessenhuis Utrecht, Utrecht, The Netherlands ${ }^{3}$ Department of Gastroenterology, Diakonessenhuis Utrecht, Utrecht, The Netherlands ${ }^{4}$ Department of Rheumatology, Diakonessenhuis Utrecht, Utrecht, The Netherlands

\section{Correspondence to} Dr Regina W Hofland, Department of Pulmonology and Tuberculosis,

Diakonessenhuis Utrecht, Bosboomstraat 1, Utrecht 3582 KE, The Netherlands; reginahofland@hotmail.com

Received 7 November 2012 Revised 9 March 2013 Accepted 14 March 2013 Published Online First 18 April 2013

\section{Tuberculosis during TNF- $\alpha$ inhibitor therapy, despite screening}

\author{
Regina W Hofland, ${ }^{1}$ Steven F T Thijsen, ${ }^{2}$ Marc A M T Verhagen, ${ }^{3}$ Yolande Schenk, ${ }^{4}$ \\ Ailko W J Bossink ${ }^{1}$
}

\section{SUMMARY}

As part of a prospective study on the safety of TNF- $\alpha$ inhibitor therapy after screening for and treatment of latent tuberculosis infection (LTBI), we report two patients who developed active tuberculosis (TB) infection during TNF- $\alpha$ inhibitor therapy, despite negative screening for LTBI. The clinical history is suggestive of a primary infection acquired during travelling to TB-endemic countries. In this lesson of the month we would like to highlight the risk of travelling to TBendemic areas in patients treated with TNF- $\alpha$ inhibitor therapy.

Screening for latent tuberculosis infection is not enough to prevent tuberculosis in patients treated with TNF- $\alpha$ inhibitor therapy

\section{CASE 1}

A 48-year-old woman suffering from inflammatory bowel disease was considered for TNF- $\alpha$ inhibitor therapy. Screening for LTBI with Tuberculin Skin Test (TST) was negative and the Interferon Gamma Release Assay T-SPOT.TB (Oxford Immunotec, Oxford, UK), was indeterminate. No prophylactic therapy was prescribed. Because of an unsatisfactory therapeutic effect, TNF- $\alpha$ inhibitor treatment (infliximab) was discontinued after 6 weeks (three doses of $5 \mathrm{mg} / \mathrm{kg}$ ). Three months later, she travelled to a highly TB-endemic country with an incidence of 101 (83-121) TB patients per 100000 persons. ${ }^{1}$ Five weeks after she returned, she developed TB meningitis and died 2 weeks later. In this patient, a primary TB infection is likely, because of her recent travel to a TB-endemic country, and the negative screening and no risk factors for LTBI before starting TNF- $\alpha$ inhibitor treatment. Even though TNF- $\alpha$ inhibitor therapy was discontinued 3 months before travelling, she can still be considered immunocompromised because the immunosuppressive effect is probably still continuing several months after treatment with TNF- $\alpha$ inhibitor therapy. ${ }^{2}$

\section{CASE 2}

A 41-year-old man, born in Morocco, with a negative TST for LTBI after his immigration to the Netherlands, developed ankylosing spondylitis and was a candidate for TNF- $\alpha$ inhibitor treatment. Therefore, LTBI screening was repeated. Because of negative TST and negative T-SPOT.TB, no prophylactic therapy was given. Seven months after starting TNF- $\alpha$ inhibitor therapy (infliximab), he travelled to Morocco, a highly TB-endemic country, for 6 weeks. Three months after he came back, he was diagnosed with extrapulmonary Mycobacterium Bovis infection. Again, a primary infection acquired during travelling is more probable than reactivation of Mycobacterium Bovis, because of repeated negative screening before TNF- $\alpha$ inhibitor treatment and the 7-month-period of therapy without signs of reactivation. It is unlikely that he is infected in the Netherlands, because active Mycobacterium Bovis infection is rare in this country.

\section{DISCUSSION}

Screening for LTBI and prophylactic treatment of LTBI is recommended before starting TNF- $\alpha$ inhibitor therapy because of risk for TB during this immunosuppressive therapy. ${ }^{3-6}$ Safety of TNF- $\alpha$ inhibitor therapy after screening for LTBI remains an important concern. There is no literature about repeated screening during TNF- $\alpha$ inhibitor therapy.

In a prospective study on the safety of TNF- $\alpha$ inhibitor therapy after screening for LTBI and treatment of LTBI, a total of 180 patients, who were considered for TNF- $\alpha$ inhibitor therapy, were included for screening. Of these patients, 144 eventually started with TNF- $\alpha$ inhibitor therapy. In the other 36 patients, TNF- $\alpha$ inhibitor therapy was not initiated because of stabilisation or improvement of the underlying disorder. Patient characteristics are described in table 1.

The screening procedure included TST and T-SPOT.TB. Before initiating TNF- $\alpha$ inhibitor therapy, 11 patients $(8 \%)$ were diagnosed with LTBI and received prophylactic therapy; 9 of them had risk factors for LTBI at screening (defined as: (1) born in a highly TB-endemic country, (2) travelling history to highly TB-endemic countries and/or (3) documented TB exposure). Follow-up varied from 3-60 months, with a mean of 25 months. Six patients $(4 \%)$ were lost to follow-up. None of the patients who received prophylactic therapy for LTBI developed active TB during follow-up. Two people, as described above, developed a new, extrapulmonary TB infection, despite the screening programme.

To the best of our knowledge, this lesson of the month is the first one reporting the development of active and primary $\mathrm{TB}$ infection during $\mathrm{TNF}-\alpha$ inhibitor treatment, despite screening, and this was related to travelling to TB-endemic areas. The immunocompromised condition attributable to TNF- $\alpha$ inhibitor therapy makes these patients susceptible to primary TB infection, usually with an extrapulmonary localisation, and can lead to serious morbidity. ${ }^{3-6}$ In the population studied, 2 (6\%) out of 36 patients who travelled to a highly TB-endemic 


\section{Table 1 Patient characteristics}

\begin{tabular}{ll}
\hline Subjects, No & 144 \\
Male, No (\%) & $63(44 \%)$ \\
Age, mean (range), years & $51(18-88)$ \\
BCG vaccinated, No (\%) & $8(6 \%)$ \\
At risk for LTBI, No (\%) & $76(53 \%)$ \\
\hline LTBI, latent tuberculosis infection.
\end{tabular}

country during, or after recent, TNF- $\alpha$ inhibitor therapy developed extrapulmonary TB. Cumulative exposure-time in highly endemic areas of all 36 patients was 158 weeks. Extrapolation of these data suggests that 79 weeks of exposure results in one case of active TB. In addition, the risk of active TB infection per year of exposure would be $66 \%$.

Patients treated with TNF- $\alpha$ inhibitor therapy should be aware of the risk related to travelling to TB-endemic countries. Current guidelines do not address this issue. This lesson implicates that a new screening for infection after travelling to TB-endemic countries should be considered together with intensive follow-up for early detection of symptoms suggestive of active TB infection. More research is required to strengthen this recommendation.

Acknowledgements The authors thank Anya Milne for carefully reviewing the manuscript.

Competing interests None.

Provenance and peer review Not commissioned; internally peer reviewed.

\section{REFERENCES}

1 WHO tuberculosis country profiles. http://www.who.int/tb/country/data/profiles/en/ index.html (accessed 31 Jan 2013).

2 Dixon WG, Symmons DP, Lunt M, et al. Serious infection following anti-tumor necrosis factor alpha therapy in patients with rheumatoid arthritis: lessons from interpreting data from observational studies. Arthritis Rheum 2007;56:2896-904.

3 Dutch association of rheumatology, in collaboration with different medical associations. Dutch guideline: Controlled use of biologicals in immune mediated inflammatory disorders. 2011. http://www.nvalt.nl/service/richtlijnen (accessed 31 Jan 2013).

4 Wallis RS. Tumour necrosis factor antagonists: structure, function, and tuberculosis risks. Lancet Infect Dis 2008:8:601-11.

5 Centers for Disease Control and Prevention (CDC). Tuberculosis associated with blocking agents against tumor necrosis factor-alpha—California, 2002-2003. MMWR Morb Mortal Wkly Rep 2004;53:683-6.

6 Association for practical tuberculosis management. Dutch guideline: IGRA in diagnosing tuberculosis. 2010. http://tbevidence.org/documents/guidelines (accessed 31 Jan 2013). 